\title{
The Effect of Starting the Protein-Sparing Modified Fast on Weight Change over 5 years
}

\author{
Elizabeth R. Pfoh, PhD, MPH', Gilbert Lowenthal, MD, MBA ${ }^{7,2}$, Laura Jeffers, Med, RD, $L D^{7}$, \\ Bartolome Burguera, MD, PhD ${ }^{3}$, Derrick Cetin, DO 4 , Bo Hu, PhD ${ }^{5}$, Niyati M. Gupta, MD', \\ Marven Cantave, MS, LD, RDN', and Michael B. Rothberg, MD, $\mathrm{MPH}^{7}$
}

\begin{abstract}
${ }^{1}$ Center for Value-Based Care Research, Cleveland Clinic Community Care, Cleveland Clinic, Cleveland, OH, USA; ${ }^{2}$ Department of Internal Medicine, Cleveland Clinic, Cleveland, $\mathrm{OH}, \mathrm{USA} ;{ }^{3}$ Endocrinology and Metabolism Institute, Cleveland Clinic, Cleveland, $\mathrm{OH}$, USA; ${ }^{4} \mathrm{Bariatric}$ and Metabolic Institute, Cleveland Clinic, Cleveland, OH, USA; ${ }^{5}$ uantitative Health Sciences, Cleveland Clinic, Cleveland, OH, USA; ${ }^{6} \mathrm{Case}$ Western Reserve University, Cleveland, OH, USA.
\end{abstract}

BACKGROUND: Ketogenic diets have been highlighted as a way to lose weight while experiencing reduced hunger. The protein-sparing modified fast (PSMF) induces ketosis but may be difficult to maintain.

OBJECTIVE: To track weight loss for individuals initiating PSMF versus all other diets (e.g., balanced, high protein) for up to 5 years.

DESIGN: Retrospective cohort study

PARTICIPANTS: Adults who discussed the PSMF with a clinician between 2007 and 2014

INTERVENTION: Initiating the PSMF diet versus other diets

MEASURES: The main outcome was percent weight change up to 5 years. Demographic and health data were collected using electronic health records. We fit regression models including age, sex, race, insurance, new medication prescriptions, and specialist visit to identify the effect of PSMF diet on percent weight change. We grouped patients by percent weight change at each year $(\geq 5 \%$ loss, $4 \%$ loss to $4 \%$ gain, $\geq 5 \%$ gain) and used Pearson $\chi^{2}$ tests to compare proportions.

RESULTS: Of 1,403 eligible patients, 879 (63\%) started the PSMF. The PSMF group was slightly younger (52 vs. 54 years, $p<0.01$ ) and had a higher body mass index (41.9 $\mathrm{kg} / \mathrm{m}^{2}$ vs. $\left.40.4 \mathrm{~kg} / \mathrm{m}^{2}, p<0.001\right)$. In the adjusted analysis, the PSMF group averaged 3\% more weight loss than the other group over the 5-year follow-up (95\% CI - 3.5, - 2.0, $p<0.001)$. PSMF patients lost more weight initially, but by year 4 , there was no difference between diets ( $1.6 \%$ versus $1.3 \%$, PSMF versus other diets, $p=0.12$ ). Patients starting the PSMF were more likely to experience $\geq 5 \%$ weight loss at 1 year (55\% vs $20 \%, p<0.001$ ) and 3 years (33\% vs. $23 \%$ $p<0.05$ ), but not 5 years (34\% vs $29 \%, p=0.16$, PSMF versus other diets, respectively).

CONCLUSIONS: In clinical practice, the PSMF achieves rapid weight loss in the first 6 months, but only a small percentage of patients maintained significant weight loss long term.

Electronic supplementary material The online version of this article (https://doi.org/10.1007/s11606-019-05535-0) contains supplementary material, which is available to authorized users.

Received February 25, 2019

Revised August 13, 2019

Accepted October 15, 2019

Published online January 8, 2020
KEY WORDS: diet; obesity; weight; primary care.

$\mathrm{J}$ Gen Intern Med 35(3):704-10

DOI: $10.1007 / \mathrm{s} 11606-019-05535-0$

(C) Society of General Internal Medicine 2020

\section{INTRODUCTION}

Low-carb, high-fat diets or ketogenic "keto" diets have been touted in the scientific and lay press as an effective way to lose weight. ${ }^{1,2}$ Individuals on low-carbohydrate diets may experience reduced hunger through achieving ketosis ${ }^{3,4}$ which occurs when the human body burns stored fat (generating ketone bodies) because calories from glucose are unavailable. ${ }^{5}$ While both low-carbohydrate and low-fat diets have been shown to reduce weight, ${ }^{6}$ reduced hunger is associated with weight loss. ${ }^{7}$

The protein-sparing modified fast (PSMF) is a very-lowcalorie, high-protein diet that aims to preserve lean body mass during weight loss by having the patient ingest $1.2-1.5 \mathrm{~g}$ of protein per day per kilogram of body weight. ${ }^{8,}{ }^{9}$ Individuals who follow the PSMF achieve ketosis, but the PSMF differs from other keto diets because the main source of calories is protein, not fat. ${ }^{910}$ Individuals who adhere to the PSMF often experience rapid and substantial weight loss, which may be attractive to patients and clinicians. ${ }^{11,12}$ However, the PSMF is not meant to be maintained long term. After approximately 6 months, individuals transition into a refeeding phase, in which they slowly reintroduce more calories and carbohydrates into their diet. Longer-term outcomes associated with the diet are less well established - studies either have time frames less than 24 months $^{13}$ or have a very small sample size. ${ }^{14}$

Understanding the weight loss trajectory of a large sample of patients following the PSMF can provide important information for clinicians and for patients who are considering the diet. The objective of this study was to track weight change for individuals who initiated the PSMF compared to patients who considered the PSMF but initiated another diet, for up to 5 years. We also assessed change in systolic and diastolic blood pressure among patients with initially elevated blood pressure $(\geq 140 / 80 \mathrm{~mm}$ of mercury ( $\mathrm{mmHg}$ )) to identify the effect of the diets on participants' cardiovascular health. 


\section{METHODS}

\section{Study population}

This retrospective cohort study included adults who received care at a large integrated health system in Northeast Ohio. Since the PSMF is an aggressive weight loss option and may select for more motivated patients, we chose as a comparison group patients who considered the PSMF diet but did not initiate it. Patients were included if they discussed starting the PSMF diet or had a uric acid laboratory value ordered within 30 days following a registered dietician (RD) visit between January 1, 2007, and December 31, 2014. Since the PSMF diet could precipitate gouty attacks, all patients who start on the PSMF diet should have a uric acid laboratory value, though that was not always the case. We searched the electronic health record for the terms "PSMF" and "protein sparing" to identify patients who discussed starting the diet. Patients who were ineligible to start the diet were excluded. This included patients who had a body mass index $(\mathrm{BMI})<30 \mathrm{~kg} / \mathrm{m}^{2}$ or an estimated glomerular filtration rate $(\mathrm{eGFR})<60 \mathrm{~mL} / \mathrm{min} / 1.73 \mathrm{~m}^{2}$ or had a diagnosis of pregnancy, chronic kidney disease, or cardiac arrhythmia or a diagnosis of any of the following diseases within 6 months prior to the RD visit: myocardial infarction, ischemic stroke, acute decompensated heart failure, liver failure, malignancy, or major psychiatric illness. We also excluded individuals who did not have a follow-up weight after the index date (i.e., the date a diet was discussed). Finally, because some patients initiate the PSMF in preparation for bariatric surgery, patients were excluded if they had bariatric surgery within 6 weeks of starting the PSMF diet. Patients who underwent bariatric surgery later were censored at the surgery date.

We then conducted a chart review to identify which patients began following the PSMF. Patients who initiated the diet constituted the intervention group. The remaining patients comprised the "other diets" group. This approach was taken to ensure a similar control group in respect to eligibility for the diet.

Our study design employed an intention-to-treat approach. Thus, all patients who initiated the PSMF diet remained in the intervention group for the entire follow-up period regardless of whether he/she completed the diet. In a sensitivity analysis, we compared individuals in the PSMF group who received at least one prescription for potassium within the first 6 months of starting the diet (a marker of being on the diet under clinician supervision) to individuals in the PSMF group who did not receive a prescription.

\section{Predictor}

The PSMF diet is a very-low-calorie diet that mainly consists of lean proteins such as chicken, seafood, and tofu. ${ }^{12}$ Calories are restricted to approximately 800 calories per day including 1.2 to $1.5 \mathrm{~g}$ of protein per kilogram per day. ${ }^{12}$ Carbohydrates are restricted to less than $20-50 \mathrm{~g}$ per day. To ensure complete nutrition, individuals on the diet need to take vitamin and mineral supplements. ${ }^{12}$ We conducted a chart review to identify the diets patients in the other-diets group stated they were on after not starting the PSMF diet.

\section{Outcomes}

The main outcome was percent weight change. We calculated the percent weight change every 3 months from baseline up to 5 years. Weight at each time point was determined by interpolating the two closest weight measurements on either side of the time point. Patients were censored after their last recorded weight or after 5 years. Our secondary outcome was change in systolic and diastolic blood pressure among patients with initial blood pressure $\geq 140 / 80 \mathrm{mmHg}$.

\section{Other variables}

To describe the population, we collected demographics (age, sex, race, insurance status), visits to an endocrinologist, antiobesity medications (e.g., Contrave, Orlistat, Topiramate, Phentermine, Saxenda, Qsymia), and antipsychotic medications, insulin, and antidepressant medications (all known to promote weight gain) from the electronic health record. We collected visits to an endocrinologist as a marker of receiving a higher intensity of care related to weight management. If a patient had at least one prescription in the year prior to starting the diet, we assumed that the medication's effect on weight was accounted for in the baseline weight and additional prescriptions were not "new." We tested this assumption by including baseline medications in the adjusted analysis. Time since initiating the diet was included as a modifier variable since the impact of the diet on weight change is anticipated to lessen over time. We modeled the regression using 90-day increments to provide the reader with finer gradations of the outcome since weight can fluctuate substantially after starting a diet.

\section{Analysis}

We used Student's $T$ test and Pearson $\chi^{2}$ tests to identify baseline differences in demographic characteristics and health care utilization between patients who started the PSMF versus another diet. We grouped patients by percent weight change at each year ( $\geq 5 \%$ loss, $4 \%$ loss to $4 \%$ gain, $\geq 5 \%$ gain) and used Pearson $\chi^{2}$ tests to compare proportions. We used a regression model adjusted for age in years, sex, race (White, Black, other), insurance status (Medicare, Medicaid, Commercial, or other), new medication prescriptions, and endocrinologist visit (yes versus no) to identify the effect of the PSMF diet type on percent weight change at each time point. To identify the effect of diet on blood pressure, we used linear regression models adjusted for demographic variables, baseline medications, BMI and blood pressure, and visits to the endocrinologist on change in systolic and diastolic blood pressure for patients who had an elevated blood pressure $(\geq 140 / 90 \mathrm{mmHg}$ ) at baseline. We reran the analysis stratified by $5 \%$ weight loss at the end of each year to identify whether the diet was independently associated with changes in blood pressure. Five percent weight loss is a 
commonly used standard for attaining health benefits association with weight loss. ${ }^{15}$ A secondary analysis identified whether achieving $\geq 3 \%$ weight loss at 3 months was predictive of weight loss across the 5-year study period. We chose the $3 \%$ threshold since prior research using data from the Look AHEAD trial found that at least $3 \%$ weight loss at 2 months was predictive of weight loss at 8 years. ${ }^{16,17}$ The sensitivity analysis reports the difference in percent weight change each year for patients with at least 1 potassium prescription in the first 6 months of starting the PSMF diet since receiving a potassium prescription was a marker of maintaining the PSMF diet.

\section{RESULTS}

The final sample included 1,403 patients, of whom $879(60 \%)$ were in the intervention group. The other group's diet approaches included a balanced diet $(36 \%)$, a high-protein diet (5\%), a standard American diet (18\%), a low-fat diet (3\%), or the diet was not stated at the next visit (39\%). At baseline, the groups were similar in sex, but the PSMF group was slightly younger and had a higher percentage of Caucasian adults (Table 1). The PSMF group had a higher initial BMI (41.8 $\mathrm{kg} / \mathrm{m}^{2}$ versus $\left.40.3 \mathrm{~kg} / \mathrm{m}^{2}, p<0.01\right)$. Forty-two percent of the PSMF group and $46 \%$ of the other-diets group had a weight recorded in year 5 (Table 2).

Table 1 Demographic Characteristics, Health Care Utilization and Outcome of Patients on the PSMF and Other Diets

\begin{tabular}{|c|c|c|c|}
\hline & $\begin{array}{l}\text { PSMF group } \\
\%(N=879)\end{array}$ & $\begin{array}{l}\text { Other-diet } \\
\text { group \% } \\
(N=\mathbf{5 2 4})\end{array}$ & $P$ value \\
\hline $\begin{array}{l}\text { Mean age in years } \\
(95 \% \text { CI })\end{array}$ & $52(52-53)$ & $54(53-55)$ & 0.01 \\
\hline Female sex $(n)$ & $79 \%(692)$ & $74 \%(387)$ & 0.06 \\
\hline Race & & & 0.02 \\
\hline White $(n)$ & $70 \%(619)$ & $64 \%(333)$ & \\
\hline Black $(n)$ & $27 \%(234)$ & $32 \%(167)$ & \\
\hline Other $(n)$ & $3 \%(26)$ & $5 \%(24)$ & \\
\hline Insurance & & & 0.03 \\
\hline Medicare $(n)$ & $10 \%(91)$ & $15 \%(79)$ & \\
\hline Medicaid $(n)$ & $5 \%(46)$ & $7 \%(35)$ & \\
\hline Commercial $(n)$ & $79 \%(698)$ & $73 \%(383)$ & \\
\hline Other $(n)$ & $5 \%(44)$ & $5 \%(27)$ & \\
\hline $\begin{array}{l}\text { Mean initial BMI in } \\
\mathrm{kg} / \mathrm{m}^{2}(95 \% \mathrm{CI})\end{array}$ & $42(41-42)$ & $40(40-41)$ & $<0.01$ \\
\hline $\begin{array}{l}\text { Mean initial weight in } \mathrm{kg} \\
(95 \% \mathrm{CI})\end{array}$ & $118(116-119)$ & $\begin{array}{l}113(111- \\
115)\end{array}$ & $<0.01$ \\
\hline \multicolumn{4}{|l|}{$\begin{array}{l}\text { Medications prescribed } \\
\text { in prior year }\end{array}$} \\
\hline Antipsychotics $(n)$ & $6 \%(49)$ & $5 \%(28)$ & 0.85 \\
\hline Antidepressants $(n)$ & $38 \%(331)$ & $29 \%(154)$ & $<0.01$ \\
\hline Weight loss $(n)$ & $13 \%(116)$ & $10 \%(52)$ & 0.07 \\
\hline Insulin $(n)$ & $6 \%(55)$ & $8 \%(39)$ & 0.39 \\
\hline \multicolumn{4}{|l|}{$\begin{array}{l}\text { Medications newly } \\
\text { prescribed in years } 1-5\end{array}$} \\
\hline Antipsychotics $(n)$ & $4 \%(39)$ & $5 \%(25)$ & 0.77 \\
\hline Antidepressants ( $n$ ) & $15 \%(129)$ & $13 \%(66)$ & 0.28 \\
\hline Weight loss $(n)$ & $19 \%(165)$ & $14 \%(74)$ & 0.03 \\
\hline Insulin $(n)$ & $8 \%(73)$ & $11 \%(55)$ & 0.17 \\
\hline $\begin{array}{l}\text { At least one visit to an } \\
\text { endocrinologist during } \\
\text { year } 1(n)\end{array}$ & $32 \%(281)$ & $19 \%(100)$ & $<0.01$ \\
\hline $\begin{array}{l}\text { Attained } 3 \% \text { weight } \\
\text { loss at } 3 \text { months }(n)\end{array}$ & $80 \%(700)$ & $26 \%$ & $<0.01$ \\
\hline
\end{tabular}

At 1 year, the PSMF group averaged 6\% more weight loss than the other-diet groups $(95 \% \mathrm{CI}, 5$ to $7 \%, p<0.01)$. The difference in percent weight loss decreased to $2 \%$ at year $2(95 \% \mathrm{CI}, 1$ to $3 \%$, $p<0.01)$ and $1 \%$ at year $3(95 \% \mathrm{CI}, 0$ to $3 \%, p=0.02)$. There was no significant difference in years 4 and 5 (Table 2). During the 5year study period, patients in the PSMF diet group averaged $3 \%$ more weight loss than the patients on other diets $(95 \%$ $\mathrm{CI},-4$ to $-2 \%, p<0.01)$ after adjusting for demographic variables, medications, and health care utilization (Fig. 1).

Percent weight change was heterogeneous within each group. One year after starting the diet, more patients in the PSMF group than the other group experienced $>5 \%$ weight loss $(55 \%$ versus $20 \%, p<0.01)$. Starting in year 2 , the percentage of patients with $>5 \%$ weight loss declined in the PSMF group and increased in the other-diets groups (38\% for PSMF versus $24 \%$ for other-diets group in year $2, p<0.001$ ). The change in weight over the entire 5-year study period appears in Fig. 2a. A similar pattern was seen for patients losing $\geq 15 \%$ of their body weight (Fig. 2b). Adjustment for potential confounders did not affect the pattern of results.

Among patients who had elevated blood pressure at baseline, adjusted systolic blood pressure dropped by an average of $8.4 \mathrm{mmHg}$ in the PSMF group and $7.2 \mathrm{mmHg}$ in the otherdiets group at year 1 compared to baseline but the difference between groups was non-significant $(p=0.24)$. Patients on the PSMF diet had a statistically greater decrease in adjusted diastolic blood pressure at year $1(-6.6 \mathrm{mmHg}$ versus -5.0 mmHG, $p=0.02$ ), but there was no statistically significant difference between groups in years 2-5. Patients who lost at least $5 \%$ of their weight in both the PSMF and other-diets group had a significantly greater change in systolic and diastolic blood pressure at year 1 than patients who lost less weight $(b=$ - $6.8 \mathrm{mmHg}$ for systolic blood pressure and $-3.9 \mathrm{mmHg}$ for diastolic blood pressure, $p<0.001$ ) (Appendix figure).

\section{Secondary and sensitivity analysis}

By 3 months, $80 \%$ of the PSMF diet group lost $\geq 3 \%$ of their body weight versus $26 \%$ of the other-diets group $(p<0.01)$. In the secondary analysis, patients who achieved early weight loss on either diet ( $\geq 3 \%$ weight loss at 3 months) averaged more weight loss than patients who did not achieve early success ( $b=6 \%, 95 \%$ CI, -7 to $-5 \%$ ) (Fig. 3 ).

In a sensitivity analysis, percent weight change among the PSMF diet group who had at least one potassium reading in the 6 months after starting the diet was higher than among those who did not, but even the latter lost substantially more weight in the first year than did the patients in the other-diets group (Appendix table).

\section{DISCUSSION}

In this retrospective cohort study, patients who started the PSMF diet averaged 3\% more weight loss over the 5-year study period than patients in the control diet. The weight loss 
Table 2 Mean percent weight change for PSMF and Control groups at years 1-5.*

\begin{tabular}{|c|c|c|c|c|c|c|}
\hline & & Year 1 & Year 2 & Year 3 & Year 4 & Year 5 \\
\hline PSMF diet & $\begin{array}{l}N \\
\text { Mean \% } \\
\text { change } \\
(95 \% \mathrm{CI})\end{array}$ & $\begin{array}{l}839 \\
-7.6(-8.3,-6.9)\end{array}$ & $\begin{array}{l}727 \\
-3.7(-4.4,-3.0)\end{array}$ & $\begin{array}{l}621 \\
-2.3(-3.0,-1.5)\end{array}$ & $\begin{array}{l}491 \\
-1.6(-2.5,-0.7)\end{array}$ & $\begin{array}{l}370 \\
-1.4(-2.5,-0.3)\end{array}$ \\
\hline Other diets & $\begin{array}{l}N \\
\text { Mean \% } \\
\text { change } \\
(95 \% \mathrm{CI})\end{array}$ & $\begin{array}{l}506 \\
-1.8(-2.3,-1.2)\end{array}$ & $\begin{array}{l}458 \\
-1.3(-2.0,-0.6)\end{array}$ & $\begin{array}{l}391 \\
-0.9(-1.7,0.0)\end{array}$ & $\begin{array}{l}312 \\
-1.1(-2.2,-0.1)\end{array}$ & $\begin{array}{l}242 \\
-1.0(-2.3,0.3)\end{array}$ \\
\hline \multirow{2}{*}{$\begin{array}{l}\text { Difference between } \\
\text { PSMF and other } \\
\text { diets }\end{array}$} & $\begin{array}{l}\text { Mean } \\
(95 \% \text { CI })\end{array}$ & $-5.8(-6.8,-4.9)$ & $-2.4(-3.4,-1.4)$ & $-1.4(-2.6,-0.3)$ & $-0.5(-1.9,0.1)$ & $-0.4(-2.1,1.3)$ \\
\hline & $p$ value & $<0.01$ & $<0.01$ & 0.02 & 0.52 & 0.64 \\
\hline
\end{tabular}

*Student $t$ tests were used to compare the mean percent weight loss between the PSMF and other-diets group at each time point

benefits of the PSMF diet were readily seen in the first year. However, the average weight loss was not statistically different across groups at year 3. Fewer than 1 in 10 patients on the PSMF diet had $\geq 15 \%$ weight loss after 5 years. Together, our findings suggest that the majority of patients who start a PSMF diet will have dramatic weight loss initially, but ultimately regain the weight. Only a small minority will succeed in maintaining meaningful weight loss.

We observed substantial heterogeneity in response to the PSMF. Twenty percent of patients who started the PSMF diet had $\mathrm{a} \geq 15 \%$ weight loss at 1 year versus only $3 \%$ of patients in the other-diets group, and early initial weight loss was associated with greater weight loss across the study period. This finding is similar to another observational study that found large initial weight loss predicted long-term weight loss. ${ }^{18}$ Moreno and colleagues found that a very-low-calorie ketogenic diet was associated with longer sustained 5\% weight loss than a standard lowcalorie diet (500 months versus 350 months). ${ }^{14}$ Our data supports this hypothesis given that in years $3-5,30 \%$ of the PSMF group maintained $\geq 5 \%$ weight loss.

These findings also suggest that some patients may gain weight by initiating the PSMF diet. The $20 \%$ of patients who started the PSMF diet and did not lose $\geq 3 \%$ in the first 3 months had a net weight gain over the study period. Our findings are similar to the Look AHEAD trial that found an association between weight loss at 2 months and long-term weight loss. ${ }^{16,17}$ Providers considering starting a patient on the PSMF diet should require a re-evaluation within the first 3 months. Another observational study found that patients on the PSMF diet who received nutritional counseling averaged $8.3 \%$ more weight loss than patients who did not receive follow-up instruction. ${ }^{13}$ Thus, following common recommendations for patients on the PSMF diet such as meeting with a

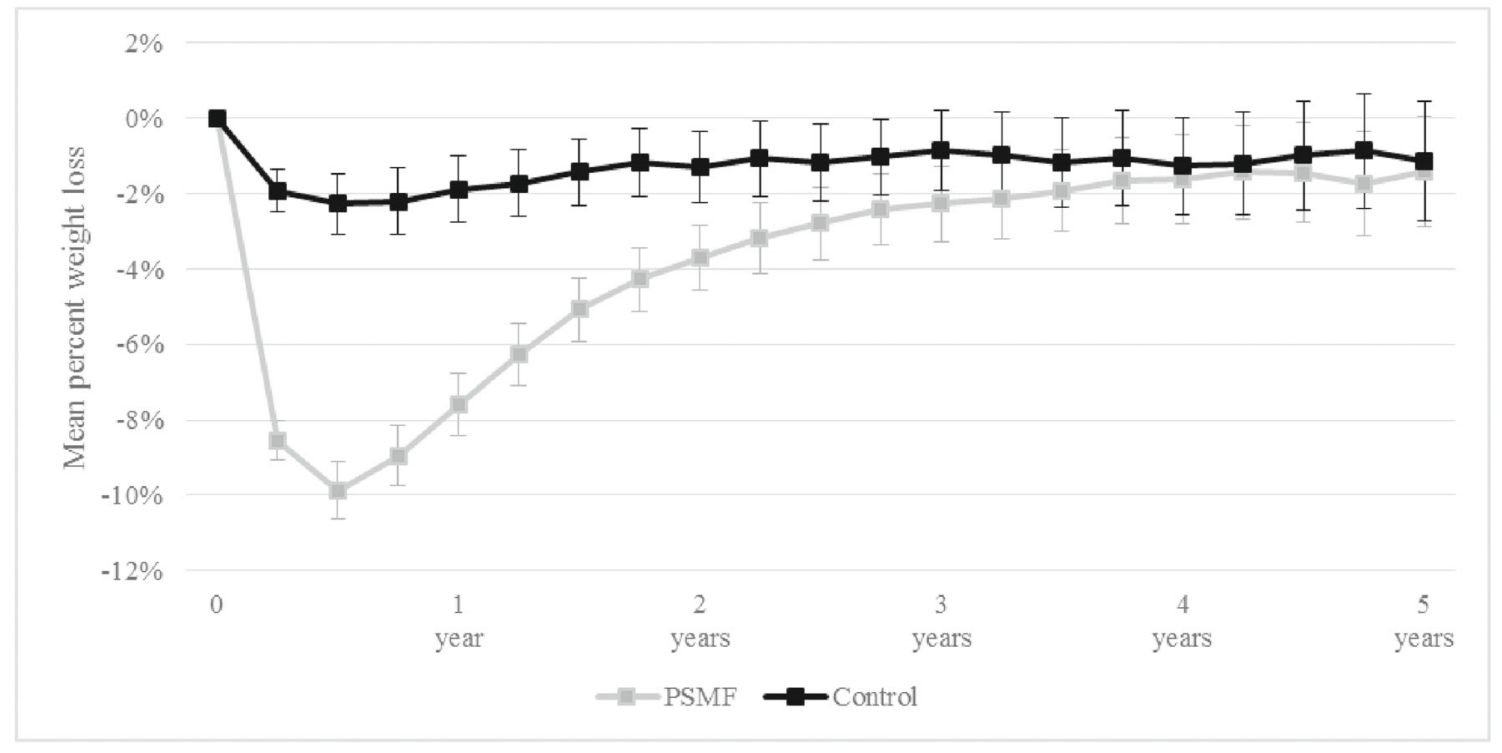

*Regression models were adjusted for age, sex, race, insurance status, new medication prescriptions and specialist visit to identify the average percent weight loss for each diet group. Percent weight loss is compared to baseline and reported every 3 months.

Figure 1 Average adjusted weight loss among PSMF and other-diet participants*. 

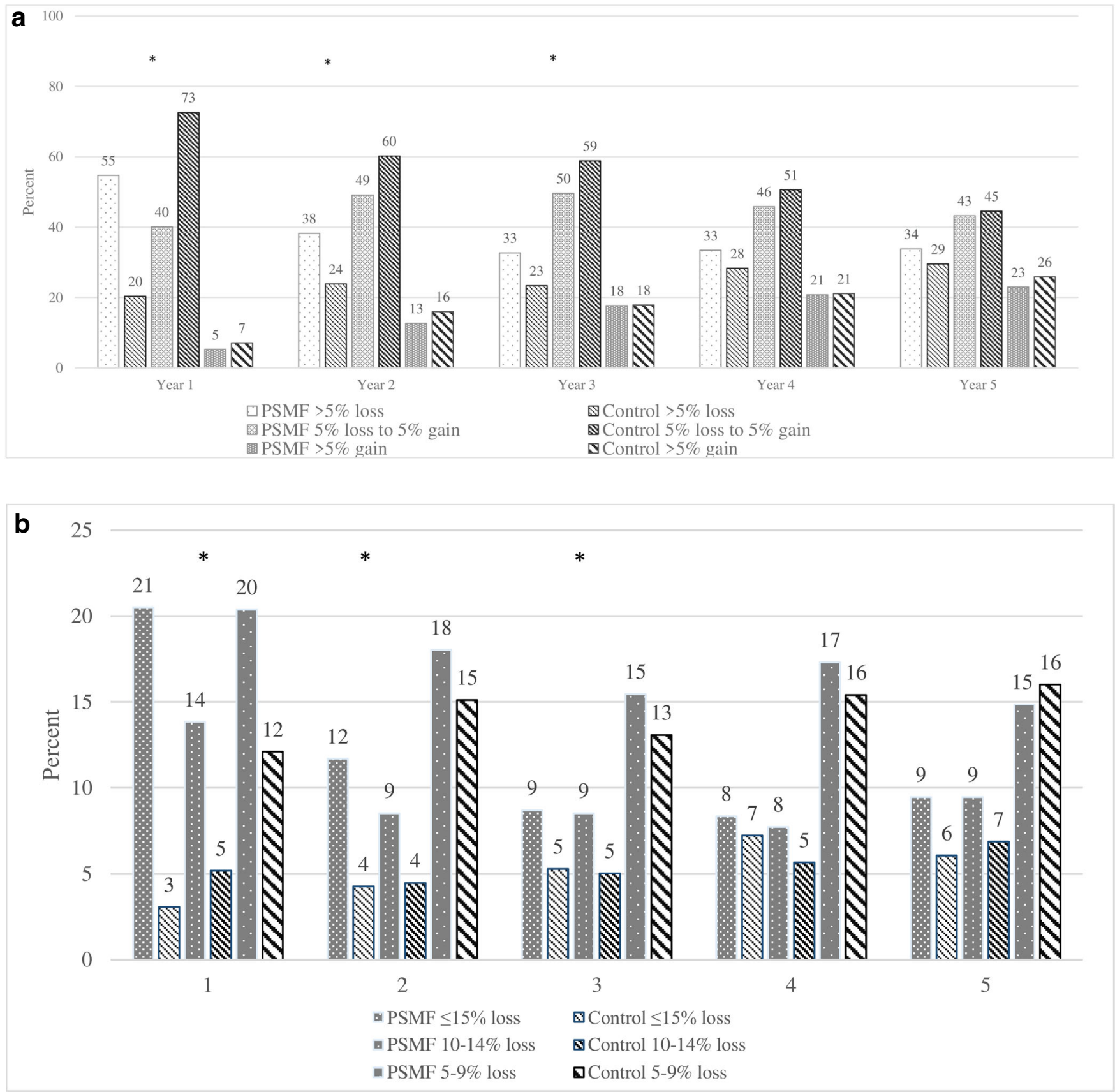

*Indicates p-values $<0.05$ comparing the effect of diet on category of percent weight loss within each year. P-values were obtained using chisquared tests.

Figure 2 a. Patients in the PSMF and control diet groups categorized into yearly percent weight loss. b. Patients in the PSMF and control diet groups who lost at least $5 \%$ of their weight in any year.v

dietician, having a basic metabolic panel and electrocardiogram, and taking a uric acid test while in ketosis may help patients to adhere to a diet in a safe and effective manner. Further, routine check-ins can allow both the clinician and patient to discuss whether the diet fits the patient's food and lifestyle preferences since it is difficult to eat the right types of food in the right quantity. ${ }^{19}$ As identified by a randomized control trial, adherence to a low-calorie diet, regardless of whether fat or protein was emphasized was associated with similar weight loss and diet satisfaction score at 2 years. ${ }^{6}$
Finally, understanding the impact of a 5\% weight loss for a couple of years may be important when considering whether to recommend the PSMF diet. Over half of the patients on the PSMF diet lost $5 \%$ of their weight at 1 year which is an often cited marker of reduced cardiovascular risk. By year 5, that proportion had dropped to one third and was similar to the proportion of patients in the other-diets group who lost $5 \%$ of their weight. For the patients who regained weight, it is unknown what cardiovascular benefit achieving a $5 \%$ weight loss at 1 year provided. In our study, blood pressure outcomes 


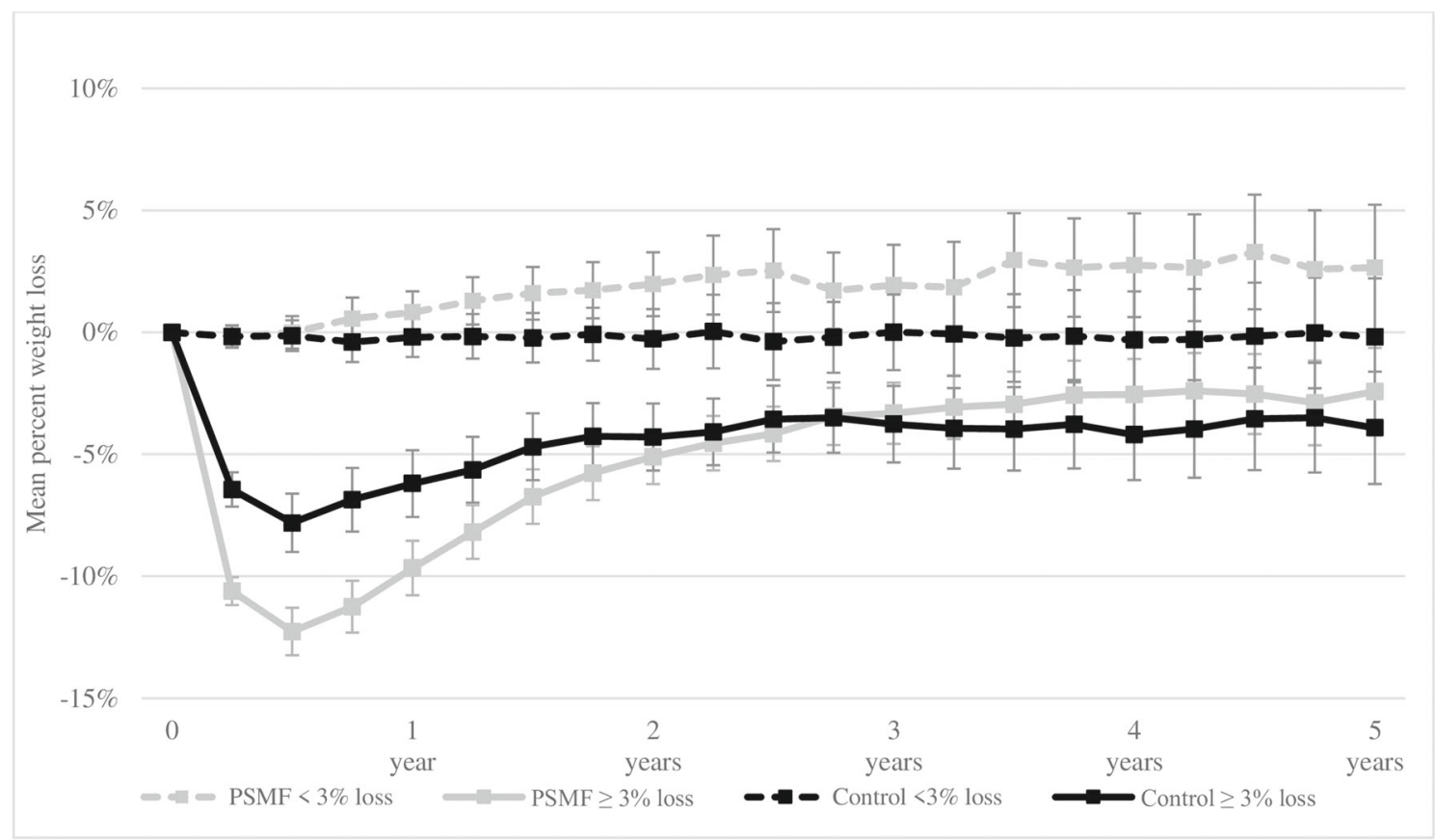

*Analysis was stratified by individuals who lost $\geq 3 \%$ of their baseline weight at 3 months. Regression models were adjusted for age, sex, race, insurance status, new medication prescriptions and specialist visit to identify the average percent weight loss for each diet group. Percent weight loss is compared to baseline and reported every 3 months.

Figure 3 Average adjusted weight loss among PSMF and other-diet participants stratified by early attainment of $3 \%$ weight loss (at 3 months).

improved for patients in both groups who lost $\geq 5 \%$ of their weight with essentially no difference between the two groups. Further, we did not require patients to stay on the PSMF diet for any specific duration, because we wanted to assess effectiveness of starting the diet, not efficacy of maintaining the diet.

This study has several limitations. Since this was a retrospective cohort study, patients who started the PSMF diet likely differed from those who did not. Further, we did not require patients to stay on the PSMF diet for any specific duration, because we wanted to assess effectiveness, not efficacy. We hope this will provide readers with relevant information regarding what to expect when recommending the PSMF diet. This study was conducted in a single large integrated health system in Ohio so generalizability may be limited. Additionally, we attempted to control for the effect of provider encouragement on weight loss by including endocrinologist visits in our adjusted model; however, residual confounding may have remained. Finally, we were only able to follow $\sim 46 \%$ of the population for 5 years. However, the drop-out rates in both the PSMF and control diet groups were similar.

In conclusion, patients who started the PSMF experienced rapid weight loss within the first year. However, most patients regained the weight and average weight loss was similar to other diets after 4 years. Although some patients succeed in maintaining their weight loss out to 5 years, a similar percentage of patients on other diets achieved similar success. Further study is needed to understand which patients will be successful with the PSMF.

Corresponding Author: Elizabeth R. Pfoh, PhD, MPH; Center for Value-Based Care Research, Cleveland Clinic Community Care, Cleveland Clinic, Cleveland, OH 44195, USA (e-mail: Pfohe@ccf.org).

\section{Compliance with Ethical Standards:}

Conflict of Interest: The authors declare that they do not have a conflict of interest.

\section{REFERENCES}

1. Abbasi J. Interest in the Ketogenic Diet Grows for Weight Loss and Type 2 Diabetes. JAMA 2018;319:215-7.

2. Ketogenic' Diet is Currently the Most Popular Weight Loss Trend-Here's Why. Cosmopolitan, 2017. 2018, at https://www.cosmopolitan.in/life/ features/a1 1523/ketogenic-diet-currently-most-popular-weight-losstrend-heres-why.) Accessed October 4, 2019

3. Ludwig DS. Lifespan Weighed Down by Diet. JAMA 2016;315:2269-70.

4. Gibson AA, Seimon RV, Lee CM, et al. Do ketogenic diets really suppress appetite? A systematic review and meta-analysis. Obes Rev 2015;16:6476.

5. Ludwig DS, Ebbeling CB. The Carbohydrate-Insulin Model of Obesity: Beyond "Calories In, Calories Out". JAMA Intern Med 2018;178:1098-103.

6. Sacks FM, Bray GA, Carey VJ, et al. Comparison of weight-loss diets with different compositions of fat, protein, and carbohydrates. N Engl J Med 2009;360:859-73.

7. Batra P, Das SK, Salinardi T, et al. Eating behaviors as predictors of weight loss in a 6 month weight loss intervention. Obesity (Silver Spring) 2013;21:2256-63. 
8. Bistrian BR. Clinical use of a protein-sparing modified fast. JAMA 1978;240:2299-302.

9. Thomas DD, Istfan NW, Bistrian BR, Apovian CM. Protein sparing therapies in acute illness and obesity: a review of George Blackburn's contributions to nutrition science. Metabolism 2018;79:83-96.

10. Bakhach M, Shah V, Harwood T, et al. The Protein-Sparing Modified Fast Diet: An Effective and Safe Approach to Induce Rapid Weight Loss in Severely Obese Adolescents. Glob Pediatr Health 2016;3:2333794X15623245.

11. Wadden TA, Stunkard AJ, Day SC, Gould RA, Rubin CJ. Less food, less hunger: reports of appetite and symptoms in a controlled study of a protein-sparing modified fast. Int $J$ Obes 1987;11:239-49.

12. Palgi A, Read JL, Greenberg I, Hoefer MA, Bistrian BR, Blackburn GL. Multidisciplinary treatment of obesity with a protein-sparing modified fast: results in 668 outpatients. Am J Public Health 1985;75:1190-4.

13. Chang JJ, Bena J, Kannan S, Kim J, Burguera B, Kashyap SR. Limited Carbohydrate Refeeding Instruction for Long-Term Weight Maintenance Following a Ketogenic, Very-Low-Calorie Meal Plan. Endocr Pract 2017;23:649-56

14. Moreno B, Crujeiras AB, Bellido D, Sajoux I, Casanueva FF. Obesity treatment by very low-calorie-ketogenic diet at two years: reduction in visceral fat and on the burden of disease. Endocrine 2016;54:681-90.

15. Blackburn G. Effect of degree of weight loss on health benefits. Obes Res 1995;3 Suppl 2:211 s-6 s.
16. Unick JL, Hogan PE, Neiberg RH, et al. Evaluation of early weight loss thresholds for identifying nonresponders to an intensive lifestyle intervention. Obesity (Silver Spring) 2014;22:1608-16.

17. Unick JL, Neiberg RH, Hogan PE, et al. Weight change in the first 2 months of a lifestyle intervention predicts weight changes 8 years later. Obesity (Silver Spring) 2015;23:1353-6.

18. Thomas JG, Bond DS, Phelan S, Hill JO, Wing RR. Weight-loss maintenance for 10 years in the National Weight Control Registry. Am J Prev Med 2014;46:17-23.

19. Denke MA. Changing Dietary Habits and Improving the Healthiness of Diets in the United States. JAMA 2016;315:2527-9.

20. graph box- Box plots.Available at: https://www.stata.com/manuals13/g2graphbox.pdf. Accessed October 4, 2019

Publisher's Note Springer Nature remains neutral with regard to jurisdictional claims in published maps and institutional affiliations. 